\title{
Dinâmica da Digitação Aplicada à Autenticação Periódica de Usuários em Ambientes Virtuais de Aprendizagem
}

\author{
Title: Keystroke Dynamics Applied to Periodic Authentication in Virtual Learning \\ Environments
}

\author{
Marco Aurélio da Silva Cruz \\ Seção de Ensino de Engenharia da \\ Computação SE/8 - Instituto Militar \\ de Engenharia (IME) - Rio de \\ Janeiro, RJ - Brasil \\ marco.aurelio.s.cruz@gmail.com
}

\author{
Julio Cesar Duarte \\ Seção de Ensino de Engenharia da \\ Computação SE/8 - Instituto Militar \\ de Engenharia (IME) - Rio de \\ Janeiro, RJ - Brasil \\ duarte@ime.eb.br
}

\author{
Ronaldo Goldschmidt \\ Seção de Ensino de Engenharia \\ da Computação SE/8 - Instituto \\ Militar de Engenharia (IME) - \\ Rio de Janeiro, RJ - Brasil \\ ronaldo.rgold@ime.eb.br
}

\begin{abstract}
Resumo
A autenticação de usuário nos ambientes virtuais de aprendizagem (AVAs) existentes é, em geral, pontual e intrusiva, ocorrendo no momento em que o usuário se conecta ao ambiente, mediante a digitação de uma senha. Tal abordagem permite, após a autenticação inicial, que usuários não credenciados assumam o papel de usuários credenciados e realizem tarefas no ambiente, causando, entre outros problemas, distorções na percepção sobre o desempenho acadêmico dos estudantes. O objetivo deste trabalho é, então, propor um mecanismo que realize a autenticação periódica não intrusiva de usuários em AVAs. O mecanismo proposto utiliza técnicas de aprendizado de máquina para construir modelos de reconhecimento baseados na dinâmica da digitação dos usuários e é independente do AVA utilizado. Um protótipo do mecanismo proposto foi implementado, integrado ao Moodle, e aplicado em uma turma de pós-graduação com dezessete usuários. Os modelos de reconhecimento gerados pelo protótipo no estudo de caso apresentaram desempenho acima de $92 \%$ de acurácia, fornecendo um indicativo favorável acerca da viabilidade de utilização do mecanismo proposto.
\end{abstract}

Palavras-Chave: dinâmica da digitação, Ambientes Virtuais de Aprendizagem, reconhecimento de usuário, Moodle

\begin{abstract}
The authentication of users on a Virtual Learning Environment (VLE) is, in general, punctual and intrusive, occurring when the user connects to the environment, by typing his password. Such approach allows, after the initial login, that unauthenticated users take the role of authenticated users and perform tasks in the environment, causing, among other things, distortions in the perception about the academic performance of students. The objective of this work is, thus, to propose a mechanism to execute periodic and non-intrusive authentications of users in VLEs. The proposed mechanism uses machine learning techniques to build recognition models based on the keystroke dynamics of users and it is also independent of the used VLE. A prototype of the proposed mechanism, integrated with Moodle, was implemented and applied to a postgraduate course with seventeen users. The recognition models generated by the prototype in the case study showed a performance above $92 \%$ of accuracy, which is a positive indication about the viability of the utilization of the proposed mechanism.
\end{abstract}

Keywords: keystroke dynamics, Virtual Learning Environments, user recognition, Moodle

Cite as: Cruz, M. A. S., Duarte, J. S. \& Goldschmidt, R. R. (2017). Keystroke Dynamics Applied to Periodic Authentication in Virtual Learning Environments (Dinâmica da Digitação Aplicada à Autenticação Periódica de Usuários em Ambientes Virtuais de Aprendizagem). Brazilian Journal of Computers in Education (Revista Brasileira de Informática na Educação - RBIE), 25(2), 36-60. DOI: 10.5753/RBIE.2017.25.02.36 


\section{Introdução}

A Educação a Distância (EaD) é uma modalidade de ensino que vem crescendo significativamente nos últimos anos em todo o mundo (Clark \& Mayer, 2016). O censo sobre a Educação superior brasileira realizado em 2015 pelo Instituto Nacional de Estudos e Pesquisas Educacionais Anísio Teixeira (INEP) revela que o número de matrículas em cursos de graduação a distância de instituições públicas e privadas cresceu, respectivamente, $16,3 \%$ e $41,2 \%$, entre 2013 e 2014 (INEP, 2014).

A evolução das tecnologias da informação e comunicação (TICs) em muito tem contribuído para apoiar o amadurecimento da EaD (Mandaji, 2012). Entre tais TICs, estão os chamados Ambientes Virtuais de Aprendizagem (AVAs). Segundo Galafassi, F. P., Gluz e Galafassi, C. (2013), AVAs são "tecnologias integradoras e abrangentes capazes de organizar e definir um ponto focal para os processos educacionais mediados por computador, apoiar os professores e promover o engajamento dos alunos". Em geral, os AVAs oferecem recursos síncronos e assíncronos para que estudantes e tutores possam interagir, independentemente da disponibilidade de tempo e da localização física de cada um.

Atualmente, existem diversos AVAs que podem ser adotados sem custo pelas instituições de ensino (Bucci \& Meneghel, 2008). Entre os mais conhecidos no Brasil estão o Moodle, o Teleduc e o AulaNet (Galafassi et al., 2013). Parte deles se mantém em constante atualização, procurando suprir demandas de seus usuários nos mais variados aspectos tais como portabilidade, usabilidade, segurança, dentre outros (Poersch, Santos, \& Nelson, 2006).

Apesar de todos os avanços proporcionados pelos AVAs na EaD, uma das principais questões acerca desta modalidade de ensino é quanto à autenticidade dos usuários dessas plataformas (King \& Case, 2014; Moten, Fitterer, Brazier, Leonard, \& Brown, 2013; Rabuzin, Baca, \& Sajko, 2006). Em geral, a autenticação de um usuário junto a um AVA é intrusiva ${ }^{1}$ e pontual, ocorrendo no momento em que o usuário se conecta ao ambiente, mediante a digitação de uma senha (Marais, Argles, \& Solms, 2006; Moini \& Madni, 2009; Rabuzin et al., 2006). No entanto, essa abordagem permite que usuários não credenciados, após a autenticação inicial, assumam o papel de usuários credenciados, o que pode ocasionar diversos problemas, como, por exemplo: falhas de segurança (por meio de acesso não autorizado a determinados conjuntos de informações e de usuários) ou distorções sobre a percepção do desempenho acadêmico dos estudantes (ao permitir que tarefas e atividades propostas no ambiente sejam desenvolvidas por usuários diferentes daqueles que, de fato, deveriam desenvolvê-las) (Moini \& Madni, 2009; King \& Case, 2014; Moten et al., 2013; Violettas, Theodorous, \& Stephanides, 2013).

A fim de trazer maior segurança às ofertas de curso na modalidade de $\mathrm{EaD}$, mitigando os problemas mencionados anteriormente, são necessários mecanismos que tornem viável um processo de autenticação periódica e não intrusiva de usuários durante o tempo em que eles atuam nos AVAs (King \& Case, 2014; Moten et al., 2013; Violettas et al., 2013).

Diante deste cenário, o objetivo do presente trabalho é propor um mecanismo que realize a autenticação periódica e não intrusiva de usuários em ambientes virtuais de aprendizagem. $\mathrm{O}$ mecanismo proposto utiliza técnicas de aprendizado de máquina para construir modelos de reconhecimento baseados na dinâmica da digitação dos usuários e é independente do AVA utilizado, podendo ser integrado a diferentes plataformas existentes. Um protótipo do mecanismo proposto foi implementado, integrado ao Moodle, e aplicado em uma situação real de utilização deste AVA. Os modelos de reconhecimento gerados pelo protótipo no estudo de

\footnotetext{
${ }^{1}$ No contexto deste artigo, o termo "intrusivo" será utilizado para designar qualquer mecanismo de autenticação que interrompa de alguma forma o fluxo de trabalho do usuário e/ou viole a privacidade do mesmo.
} 
caso apresentaram desempenho acima de $92 \%$ em termos de acurácia, fornecendo um indicativo favorável acerca da viabilidade do mecanismo proposto.

Este texto encontra-se organizado em mais seis seções. A Seção 2 apresenta os conceitos básicos sobre a dinâmica da digitação e o aprendizado de máquina, necessários à compreensão do mecanismo de autenticação periódica de usuário proposto. A Seção 3 discute alguns dos principais trabalhos relacionados, comparando-os com o mecanismo proposto. $\mathrm{O}$ funcionamento de cada uma das etapas da abordagem seguidas pelo mecanismo proposto encontra-se descrito na Seção 4. A Seção 5 indica os detalhes do protótipo que implementa a abordagem proposta. Detalhes sobre o estudo de caso realizado, os experimentos desenvolvidos e os resultados obtidos são apresentados na Seção 6. A Seção 7 finaliza o trabalho, resumindo as principais conclusões e expondo possibilidades de trabalhos futuros.

\section{Fundamentos}

\subsection{Reconhecimento de Usuário Baseado em Dinâmica da Digitação}

Existem basicamente três formas de se autenticar usuários em sistemas de informação (O’Gorman, 2003):

- a partir de recursos de que o usuário disponha (e.g. a utilização de tokens);

- a partir de informações conhecidas pelo usuário, como, por exemplo, o uso de senha (uma sequência de caracteres previamente memorizada), sendo esta a forma de autenticação mais comumente utilizada;

- a partir dos atributos físicos ou das características biométricas do usuário.

Existem diversas técnicas biométricas que exploram diferentes partes e características físicas do corpo, como, por exemplo, a análise da face, da íris dos olhos, da digital dos dedos, dentre outras (Jain, Nandakumar, \& Ross, 2016).

A dinâmica da digitação é uma técnica biométrica que busca identificar pessoas por meio do seu padrão de digitação (Alsultan \& Warwick, 2013). Segundo essa técnica, na medida em que o usuário digita informações no teclado, são coletados dados em sua forma bruta (denominação adotada neste artigo para indicar dados que não tenham recebido nenhum tratamento especial). Tais dados são armazenados em uma estrutura simples, tal como a indicada na Tabela 1 e compreendem o código da tecla pressionada, o tempo (escrito como uma sequência de números que representa a quantidade de milissegundos desde primeiro de janeiro de 1970 (ECMA, 2015) até o momento da ação), e a ação, que é o tipo de evento ocorrido.

Tabela 1: Estrutura dos Dados Brutos

\begin{tabular}{|c|c|c|}
\hline Tecla & Tempo & Ação \\
\hline 65 & 21469976126324 & Down \\
\hline 65 & 21469976126430 & Up \\
\hline 70 & 21469976126525 & Down \\
\hline 70 & 21469976126629 & Up \\
\hline
\end{tabular}

Desses dados brutos são extraídas informações, como a latência entre as teclas pressionadas e o tempo de pressionamento.

O tempo de pressionamento corresponde ao tempo em que uma tecla demorou sendo pressionada. Para conseguir esta informação, basta diminuir o instante de tempo capturado no momento em que a tecla foi solta pelo instante de tempo capturado quando a tecla foi pressionada (Banerjee \& Woodard, 2012). Por exemplo, na Figura 1 os eventos de pressionamento e soltura da tecla são representados por Down e Up, respectivamente. Portanto, 
o resultado da subtração do tempo obtido no evento Up ocorrido na tecla "B" com o de Down da mesma é igual ao tempo de pressionamento, representado por P1. O mesmo se repete para as demais teclas.

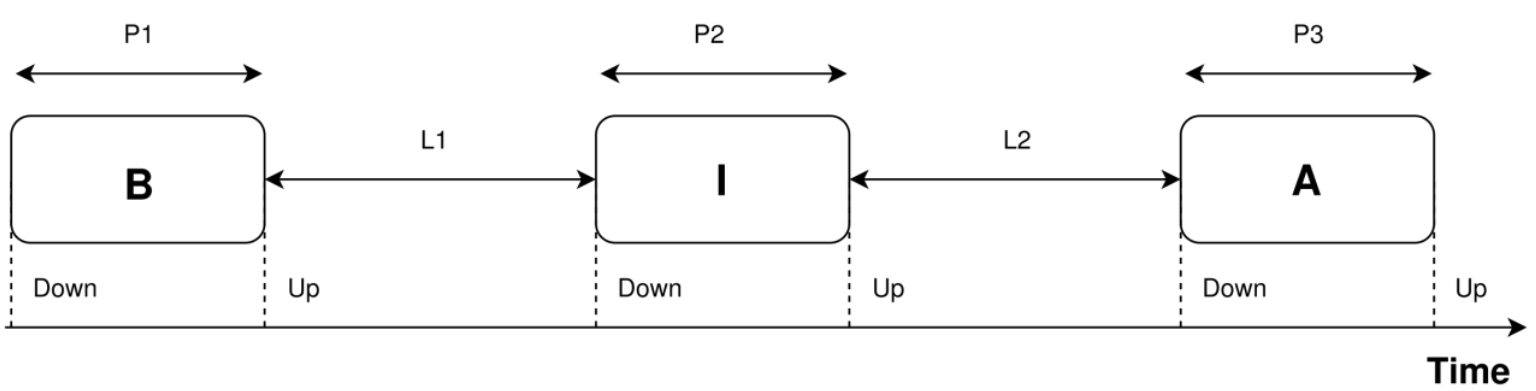

Figura 1: Processo de coleta de dados da digitação adaptado de Boechat, Ferreira, \& Carvalho Filho (2007).

A latência é o tempo entre o pressionamento de duas teclas. Ela pode ser explorada de diferentes maneiras. Por exemplo, podem ser utilizadas as distâncias entre dois eventos Down consecutivos de duas teclas, ou dois eventos Up consecutivos, entre outros. Alternativamente, pode ser utilizada a combinação de diferentes eventos (Banerjee \& Woodard, 2012; Costa et al., 2005). No exemplo da Figura 1, a latência é o tempo decorrido entre o evento Up de uma tecla com o evento Down da tecla seguinte, representada por L1 e L2. Diferentemente do tempo de pressionamento, a latência pode resultar em valores negativos, bastando uma tecla ser pressionada antes da tecla anterior a ela ser solta.

Em geral, após a coleta dos dados brutos, é executada uma fase de extração de características consideradas relevantes para o processo de reconhecimento do usuário (Costa et al., 2005; Cavalcanti, 2005; Araújo, Sucupira, Lizarraga, Ling, \& Yabu-Uti, 2005). Tal fase consiste em gerar novos atributos a partir dos dados coletados. A média e o desvio padrão do tempo de pressionamento das teclas são exemplos de informações que podem ser extraídas a partir da consolidação dos dados brutos coletados durante a digitação dos textos. Este tipo de consolidação permite construir modelos de reconhecimento que sejam independentes das teclas digitadas pelos usuários.

Uma providência comumente adotada após a extração de características dos dados coletados é a normalização dessas características (Cavalcanti, 2005). A normalização de dados consiste em ajustar os valores dos atributos de forma que todos assumam uma mesma ordem de grandeza (Goldschmidt, Bezerra, \& Passos, 2015). Tal providência é importante para evitar que alguns atributos, por apresentarem escalas de valores em ordem de grandeza maior que outros, influenciem de forma tendenciosa a construção dos modelos de reconhecimento de usuário.

Em geral, o reconhecimento de usuário é tratado como um problema de classificação, uma das mais importantes tarefas de aprendizado de máquina (Goldschmidt et al., 2015). Existem diferentes algoritmos de classificação que podem ser utilizados no reconhecimento de usuário (Faceli, Lorena, Gama, \& Carvalho, 2011), inclusive no contexto da dinâmica da digitação (Bhatt \& Santhanam, 2013; Monrose \& Rubin, 2000; Teh, Teoh, \& Yue, 2013). Detalhes sobre esses algoritmos se encontram na próxima seção.

\subsection{Aprendizado de Máquina}

No contexto de aprendizado de máquina, a tarefa de Classificação consiste em, dado um conjunto de registros de entrada associados a classes (rótulos categóricos pré-definidos), construir um modelo que seja capaz de mapear novos registros nas classes pré-definidas correspondentes (Goldschmidt et al., 2015). Em geral, a tarefa de classificação segue a 
abordagem de aprendizado supervisionado, que se divide em duas fases: uma de treinamento e outra de testes.

A fase de treinamento compreende a abstração de um modelo de conhecimento a partir dos dados apresentados na forma de pares ordenados (entrada, saída desejada). No contexto de reconhecimento de usuários baseado na dinâmica da digitação, cada par ordenado corresponde a uma amostra dos dados coletados. A entrada consiste de um conjunto de valores extraídos e normalizados a partir dos dados brutos da amostra (vide Seção 2.1). A saída desejada é uma classe entre duas possíveis: positiva (caso o texto da amostra tenha sido digitado pelo usuário correspondente) ou negativa (caso contrário). Diferentes amostras devem ser utilizadas no conjunto de treinamento, sendo a quantidade dessas amostras, das duas classes, equilibrada.

A etapa de testes tem como objetivo avaliar o desempenho do modelo de classificação produzido pela etapa anterior. Para isso, recebe como entrada um conjunto de teste formado por pares ordenados (entrada, saída desejada) diferentes dos utilizados no treinamento. A entrada de cada par é submetida ao modelo que produz uma resposta e a compara com a saída desejada, computando acerto (caso a saída desejada coincida com a saída produzida) ou erro (caso contrário). Ao final desta etapa, o desempenho do modelo é aferido.

Dentre as medidas de desempenho usualmente utilizadas para avaliar modelos de classificação, a acurácia é uma das mais populares (Goldschmidt et al., 2015). Ela informa o percentual de acertos do modelo em relação ao total de amostras do conjunto de testes. Logo, quanto maior a acurácia do modelo, melhor é o desempenho do referido modelo.

A construção, a avaliação e a aplicação de modelos de classificação é realizada por meio de algoritmos de classificação (Faceli et al., 2011).

Embora existam diversos algoritmos de classificação baseados em diferentes critérios (vieses) que orientam a construção de seus modelos, nenhum deles se mostra absolutamente melhor que todos os demais (Goldschmidt et al., 2015). Em geral, dado um cenário de aplicação, vários algoritmos de classificação são experimentados a fim de verificar sua adequação ao contexto. A seguir, é apresentada uma breve descrição dos algoritmos utilizados no presente trabalho. A escolha deste conjunto teve como objetivo diversificar os vieses de concepção dos algoritmos, trazendo diversidade ao universo de algoritmos experimentados.

O Algoritmo K-Vizinhos Mais Próximos (K-NN) (Cover \& Hart, 1967) classifica um novo registro com a classe majoritária dentre os seus $\mathrm{k}$ vizinhos mais próximos, a menos que sejam atribuídos pesos diferentes para vizinhos mais próximos, isto é, com menores distâncias. Uma métrica de distância comumente utilizada no K-NN é a distância Euclidiana.

Já no algoritmo Centroide Mais Próximo (Tibshirani, Hastie, Narasimhan, \& Chu, 2002), cada classe é representada por um centroide (médias do exemplos de treinamento), donde um novo registro é classificado como sendo da classe cujo centroide é o mais próximo. A distância é calculada utilizando uma métrica que pode ser a Euclidiana, a de Manhattan, dentre outras.

Árvores de Decisão (Salzberg, 1994) são modelos de representação do conhecimento, segundo uma estrutura de dados em árvore, onde cada nó interno representa uma decisão sobre um atributo que determina como os dados estão particionados em seus nós-filhos, até um nófolha que indica a sua classe. Para chegar a melhor classificação, em cada iteração feita na árvore, isto é, em cada divisão de uma subárvore, é escolhido o melhor atributo de acordo com uma métrica. Possíveis métricas utilizadas (Fayyad \& Irani, 1992) são a Entropia, utilizada nos algorítmos C4.5, ID3 e a medida de impureza Gini, usada no pelo algorítmo CART (Classification and Regression Tree).

O algoritmo Floresta Aleatória (Breiman, 2001), gera um conjunto de árvores de decisão que contém amostras criadas por um vetor randômico. A classe majoritária, dentre todas as 
árvores treinadas, é então designada como a classe de um exemplo. A sua acurácia depende do desempenho das suas árvores, porém, ela tende a ter melhores resultados que as árvores individuais. Além disso, uma das vantagens de sua utilização é a sua velocidade (Breiman, 2001).

O algoritmo de Máquinas de Vetores de Suporte (SVM) (Cortes \& Vapnik, 1995) constrói classificadores lineares que separam o conjunto de dados de treinamento por meio de um hiperplano representado em um hiperespaço de maior dimensão do exemplo, criado por meio de uma função-núcleo aplicada aos exemplos. Com esta função, conjuntos de dados não linearmente separáveis podem ser mapeados em um espaço de dimensão maior e com isso podem obter melhores resultados. Ao utilizar este algorítmo é importante levar em consideração a sua taxa de penalidade de erro (também chamada de $\mathrm{C}$ ). Uma alta penalidade tende a fazer o classificador elevar a quantidade de falsos-positivos.

O Classificador Bayesiano Ingênuo (Naïve Bayes) (Langley, Iba, \& Thompson, 1992) gera modelos probabilísticos, supondo que os exemplos sigam uma distribuição de probabilidade particular e que seus atributos não sejam dependentes entre si.

Por último, as Redes Neurais Artificias (Rosenblatt, 1962) constituem-se de uma técnica baseada na simulação do funcionamento do cérebro humano, em que estruturas complexas (redes) de neurônios artificiais (regressores lineares) são criadas de forma a permitir a classificação. Em linhas gerais, essas estruturas se interligam para formar uma rede com três camadas interligadas. A primeira é uma camada receptora, que é capaz de absorver os sinais externos, ou dados de entrada. Uma camada intermediária que processa os dados recebidos de diferentes maneiras (pode possuir subcamadas internas e varios neurônios). E por fim, uma camada de saída com neurônios de resposta. Os neurônios se ligam, recebendo n entradas e produzindo uma saída, ou peso. Esses pesos são ajustados durante a fase de treinamento de acordo com uma função de erro.

Uma técnica muito utilizada na comparação entre algoritmos de classificação é a validação cruzada estratificada com k-folds (Kohavi, 1995). Esta técnica divide o conjunto de amostras em $\mathrm{k}$ subconjuntos (em geral, utiliza-se $\mathrm{k}$ igual a 10), procurando equilibrar a quantidade de classes em cada subconjunto. Em seguida, executa um processo iterativo em que, a cada rodada, um dos k subconjuntos é considerado como conjunto de testes e os (k-1) restantes compõem o conjunto de treinamento. O processo se repete $\mathrm{k}$ vezes para cada algoritmo e um desempenho médio por algoritmo é calculado e usado na comparação direta entre eles.

\section{Trabalhos Relacionados}

\subsection{Reconhecimento de Usuário}

É muito comum sistemas de informação serem projetados com apenas um processo de autenticação de usuários feito no momento do primeiro acesso e com base em um conjunto de caracteres (senha) que o usuário conhece (Moini \& Madni, 2009; Rabuzin et al., 2006; Marais et al., 2006). Porém, nada impede que usuários informem suas credenciais a outras pessoas, o que compromete a fidedignidade do processo.

Diante deste cenário, existem diferentes técnicas de reconhecimento de usuários que exploram as características intrínsecas ao indivíduo, são as chamadas técnicas biométricas (Monrose \& Rubin, 2000).

Entre as principais técnicas biométricas estão o reconhecimento (Jain et al., 2016):

- da íris (Daugman, 2004; Bowyer, Hollingsworth, \& Flynn, 2013): uma técnica que se baseia na extração de características da textura da íris; ela é interessante por apresentar 
boa acurácia; necessita de equipamentos específicos para gerar imagens dos olhos e possui limitações quanto a movimentação da cabeça e da pálpebra;

- da face (Parkhi, Vedaldi, \& Zisserman, 2015; Jafri \& Arabnia, 2009): busca identificar pessoas por diferentes características ligadas a geometria da face e outras particularidades; sua precisão é boa e tem crescido, principalmente com a evolução dos dispositivos fotográficos e das técnicas de modo geral; necessita de dispositivos para capturar imagens e é intrusiva;

- da voz (Padmanabhan \& Premkumar, 2015): identifica pessoas através do padrão de voz; apresenta boa acurácia em ambientes controlados; porém, pode ter problemas com ruídos sonoros e distância do microfone, além de ser intrusiva;

- da digital dos dedos e mãos (Ali, Mahale, Yannawar, \& Gaikwad, 2016; Maio, Maltoni, Cappelli, Wayman, \& Jain, 2002): o reconhecimento de digitais é amplamente utilizado; possui boa acurácia, porém, necessita de hardware específico para capturar as digitais.

Outra técnica biométrica de reconhecimento de usuário que vem ganhando notoriedade nos últimos anos é a dinâmica da digitação. Detalhes sobre ela podem ser obtidos na Seção 3.2.

\subsection{Reconhecimento de Usuários Baseado na Dinâmica da Digitação}

A digitação periódica de senhas (ou tokens) não se mostra uma alternativa viável na maioria dos casos, pois ela pode comprometer a usabilidade do sistema, uma vez que solicitações recorrentes de senha podem causar interrupções indesejadas no desenvolvimento das atividades.

Por outro lado, o uso de técnicas biométricas tais como as apresentadas na Seção 3.1 demanda a utilização de equipamentos extras, nem sempre disponíveis em todos os ambientes.

O reconhecimento de usuário baseado na dinâmica da digitação é uma solução factível para mitigar esses problemas, pois é uma técnica biométrica. Tal característica impede que os usuários possam simplesmente passar suas credenciais para outras pessoas. Além disso, não necessita de nenhum equipamento adicional para a utilização.

A técnica de reconhecimento baseada na dinâmica da digitação pode agir como uma camada de segurança adicional em aplicações com autenticações básicas (apenas login e senha), para garantir a autenticidade do usuário durante o seu uso da aplicação, seja de maneira periódica ou contínua (Dowland, Furnell, \& Papadaki, 2002).

Uma característica importante dessa técnica é que o processo de reconhecimento de usuário não é intrusivo (Banerjee \& Woodard, 2012). Assim, a pessoa pode nem mesmo notar que está sendo avaliada enquanto desempenha uma atividade de escrita no sistema. Tal característica é um fator importante para a execução de tarefas que demandem concentração. Além disso, pode contribuir para diminuir os problemas de privacidade que podem ser gerados por abordagens biométricas que reconhecem face, íris ou voz.

É importante notar que o reconhecimento de usuário a partir da análise do padrão de digitação pode ser aplicado em diferentes contextos (Alsultan \& Warwick, 2013), não sendo restrito apenas na $\mathrm{EaD}$.

As pesquisas que exploram a análise de padrões de digitação dividem-se em duas categorias: aquelas cujos padrões são identificados a partir da digitação de textos pré-definidos e aquelas que analisam padrões de digitação de textos dinâmicos (o usuário decide o que escrever) (Alsultan \& Warwick, 2013). Em geral, o reconhecimento de usuários por meio de padrões de digitação pré-definidos apresenta melhor desempenho do que pela digitação de textos dinâmicos (Gunetti \& Picardi, 2005). Isso ocorre basicamente porque os usuários escrevem sequências de caracteres previamente conhecidas, evitando que ocorram interrupções para pensamento ou consulta durante a digitação. $\mathrm{O}$ presente trabalho se enquadra na segunda categoria, uma vez 
que, normalmente não existem regras para a produção dos textos usados na comunicação em AVAs.

Várias pesquisas de reconhecimento de usuário realizadas com base na digitação de textos dinâmicos aplicadas em diferentes contextos com diferentes números de usuários (mais de 100) e de amostras (mais de 2.000) relataram bons resultados no processo de classificação, atingindo valores de acurácia acima de 97\% (Alsultan \& Warwick, 2013; Bhatt \& Santhanam, 2013; Gunetti e Ruffo, 1999; Curtin etal., 2006). Tais resultados sugerem que o uso desse tipo de abordagem no reconhecimento de usuários pode ser promissor também no contexto da EaD.

\subsection{Mecanismos de Autenticação de Usuários em AVAs}

O uso de autenticação de usuários em AVAs com apenas login e senha "aumenta a vulnerabilidade a fraudes, tanto no acesso ao sistema quanto durante a participação do aluno nas atividades do curso, pois outra pessoa pode substituir facilmente o indivíduo que deveria ser avaliado no AVA acessado" (Rabuzin et al., 2006) apud (Diniz, Neto, Júnior, \& Fontes, 2012).

A baixa de segurança decorrente do uso de senhas incentiva o desenvolvimento de soluções para tornar o ambiente educacional na modalidade a distância mais confiável. Uma solução para a EaD é o uso de técnicas biométricas para validar os usuários dos AVAs. Alguns estudos neste cenário foram realizados e seguem comentados abaixo.

Alguns trabalhos investigaram o uso de reconhecimento facial para autenticação de usuários em AVAs (Diniz et al., 2012; Penteado \& Marana, 2008; Rolim \& Bezerra, 2008). O problema para a adoção prática desta abordagem é que ela pode violar privacidade, ao revelar por meio de imagens, momentos de intimidade dos usuários.

Outros trabalhos combinaram diferentes tipos de biometria para o reconhecimento de usuários (Moini \& Madni, 2009; Dehnavi, Sharafi, \& Nematbakhsh, 2011). No trabalho de Moini e Madni (2009), foi investigada uma abordagem baseada no reconhecimento de digitais e da face. No trabalho de Dehnavi et al. (2011), foi feito reconhecimento facial, e na análise dos padrões da digitação e do mouse. Embora a combinação de diferentes tipos de biometria possa melhorar a acurácia do processo de reconhecimento, ambos os trabalhos propuseram soluções que, além de violar privacidade, geram interrupções em diferentes momentos do uso do sistema, forçando o usuário a se autenticar diversas vezes ao longo do processo. Além disso, para viabilizar a abordagem proposta por esses trabalhos, é preciso que o usuário possua uma câmera, o que nem sempre ocorre em computadores pessoais ou empresariais.

Utilizar a dinâmica da digitação no reconhecimento de usuários da $\mathrm{EaD}$ pode mitigar os problemas mencionados. O processo de reconhecimento pode ocorrer de forma periódica e transparente para o usuário. E, além disso, o único requisito de hardware necessário é um teclado, componente usualmente necessário na utilização de AVAs em geral.

Apesar do exposto, não foram encontrados trabalhos que investigassem a viabilidade do uso da abordagem de reconhecimento de usuário baseada na dinâmica da digitação aplicada de forma periódica, não intrusiva e integrada a AVAs.

\section{Mecanismo Proposto}

Conceitualmente, o processamento do mecanismo proposto se divide em três fases. A primeira é responsável por coletar amostras de digitação de usuários do AVA e permitir a construção de um modelo de reconhecimento específico para cada usuário do ambiente. A segunda fase compreende a aplicação desses modelos na medida em que os respectivos usuários utilizam as funcionalidades do AVA. A cada vez que um usuário digita algum texto em uma funcionalidade 
do ambiente, o mecanismo proposto avalia o padrão de digitação, aplicando o modelo de reconhecimento correspondente, e armazena o resultado da avaliação junto ao texto gravado. A terceira fase compreende a apresentação de relatórios periódicos que indicam usuários responsáveis por ocorrências de digitação cuja autoria não tenha sido reconhecida pelo mecanismo proposto. As figuras 2, 3 e 4 ilustram graficamente os módulos funcionais da primeira, segunda e terceira fases, respectivamente. As próximas subseções detalham cada um desses módulos funcionais.

Cabe ainda ressaltar que para que o mecanismo proposto opere de forma integrada ao AVA desejado, o administrador do sistema deve configurar o ambiente, definindo que funcionalidades deverão ser submetidas ao processo de autenticação periódica.

É importante também notar que a análise da dinâmica da digitação é complementar ao reconhecimento inicial de usuário por meio de login e senha.

\subsection{Coleta de Dados}

De forma a verificar se um usuário é realmente quem ele diz ser, usando a dinâmica da digitação, é necessário antes, capturar o padrão de digitação desse usuário. O módulo de Coleta de Dados, primeiro módulo da Figura 2, tem como objetivo capturar amostras do padrão de digitação.

Com o intuito de identificar o padrão de digitação do usuário, o módulo de Coleta de Dados apresenta um formulário em branco com perguntas cujas respostas precisam ser digitadas. Em algumas delas, o conteúdo do texto a ser digitado é fixo. Nas demais o texto é dinâmico, podendo ser digitado livremente pelo usuário.

Na medida em que o usuário preenche o formulário, os registros de digitação de cada tecla são coletados de forma transparente ao usuário e são armazenados em uma espécie de histórico, que contém os dados brutos, no formato apresentado na Seção 2.1.

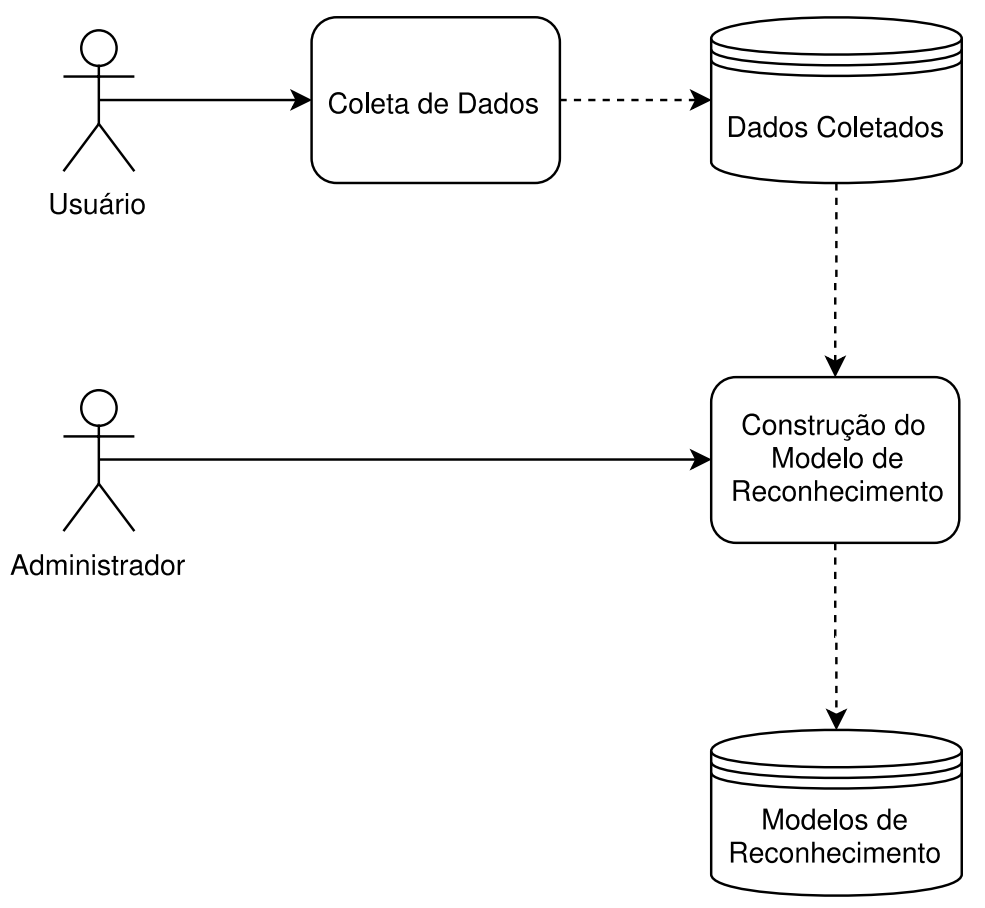

Figura 2: Primeira Fase do Mecanismo Proposto: Coleta de Dados e Construção do Modelo de Reconhecimento do Usuário. 


\subsection{Construção do Modelo de Reconhecimento}

Esta seção detalha o segundo módulo apresentado na Figura 2. Nele, um modelo de reconhecimento é construído para cada usuário do AVA. A construção de cada modelo é um processo que ocorre em quatro etapas, sob coordenação do administrador do sistema.

Uma vez escolhido um usuário do AVA, a primeira etapa do processo de construção do modelo de reconhecimento é a extração de características da digitação presente em cada amostra de texto digitada pelo referido usuário no módulo de Coleta de Dados. Tais características são: o tempo de pressionamento das teclas e o tempo de latência entre teclas.

$\mathrm{Na}$ segunda etapa, é feita a construção de atributos, em que tais características dão origem a novos atributos com informações consolidadas. Tais atributos são: a média e o desvio padrão do tempo de pressionamento das teclas e da latência entre as teclas.

$\mathrm{Na}$ etapa seguinte, os atributos consolidados são normalizados, colocando-os na mesma ordem de grandeza para efeito de comparação e melhor aplicação no algoritmo responsável pelo treinamento.

$\mathrm{Na}$ última etapa, o Administrador do Sistema aplica diversos algoritmos de aprendizado de máquina a fim de selecionar qual o mais indicado para o usuário em questão. A comparação entre os algoritmos é feita por meio de um processo de validação cruzada em k-folds, sendo a acurácia, a métrica de avaliação escolhida. O algoritmo com o melhor desempenho médio na validação cruzada é considerado o vencedor e o modelo de reconhecimento adotado é o que obteve o melhor desempenho durante o processo de validação cruzada do referido algoritmo. Tal modelo é armazenado em uma base de conhecimento de forma a ser utilizado pelo módulo de aplicação do modelo de reconhecimento, sempre que for necessária a autenticação do usuário correspondente.

\subsection{Verificação de Autoria}

Uma vez que o modelo de reconhecimento de um usuário tenha sido construído e armazenado na base de conhecimento, sempre que tal usuário acessar uma funcionalidade do AVA que esteja associada ao mecanismo proposto, o módulo de Verificação de Autoria é acionado, conforme apresentado na Figura 3. Note que a funcionalidade do AVA aparece em uma caixa de bordas pontilhadas na Figura 3, pois ela não faz parte do mecanismo proposto, devendo ser ajustada para acionar o módulo de Verificação de Autoria. O processamento deste módulo ocorre em três etapas.

Durante a primeira etapa, na medida em que o usuário digita os dados da funcionalidade do AVA, os registros de digitação de cada tecla são coletados de forma transparente ao usuário, de forma análoga ao processo realizado pelo módulo de Coleta de Dados. 


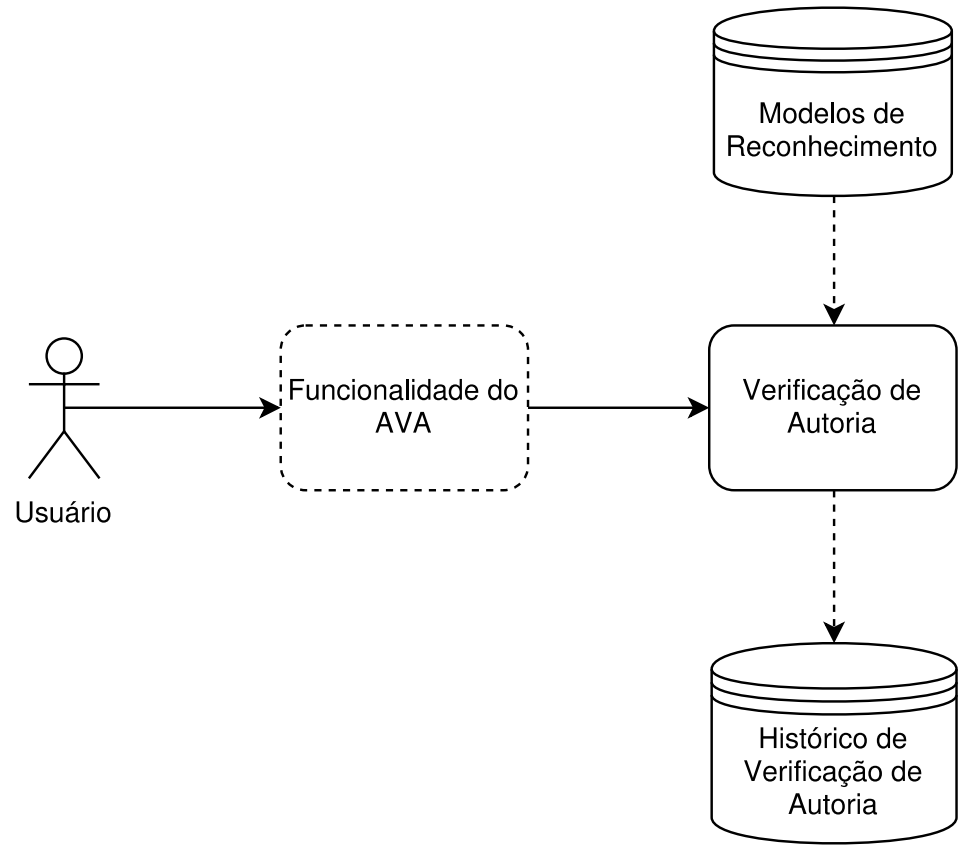

Figura 3: Segunda Fase do Mecanismo Proposto: Verificação de Autoria.

Após o usuário concluir a digitação e os dados brutos serem salvos, a segunda etapa do módulo de Aplicação do Modelo de Reconhecimento é iniciada. De forma análoga ao descrito no módulo de Construção do Modelo Preditivo, nesta etapa, são calculados o tempo de pressionamento das teclas e o tempo de latência entre teclas, que, em seguida, passam pela geração de atributos, gerando assim, a média e o desvio padrão de cada uma dessas características. Por último, assim como no treinamento, os atributos são normalizados.

$\mathrm{Na}$ última etapa, o modelo de reconhecimento do usuário que está interagindo com o AVA é recuperado da base de conhecimento e aplicado sobre os dados processados na etapa anterior, a fim de verificar se o usuário em questão foi, de fato, o autor dos dados recém digitados. Os resultados possíveis desta etapa são: autoria confirmada (classe positiva) ou autoria suspeita (classe negativa). O resultado obtido é armazenado de forma vinculada aos dados digitados e pode ser consultado pelo módulo de Análise de Autorias da terceira fase de operação do mecanismo proposto.

\subsection{Análise de Autorias}

$\mathrm{Na}$ terceira e última fase de operação do mecanismo proposto, gestores acadêmicos podem acessar os resultados das verificações de autoria realizadas durante o período em que os usuários desenvolveram suas atividades no AVA. Isto é feito por meio do módulo de Análise de Autorias, representado na Figura 4. Este módulo provê dois tipos de visão sobre os resultados: visão sintética e visão detalhada. Na visão sintética, o gestor acadêmico pode consultar os usuários do AVA pelos percentuais de autorias suspeitas e confirmadas dentro de um intervalo de tempo. Na visão detalhada, o gestor tem acesso às informações vinculadas a cada resultado, podendo visualizar, por exemplo, que tipo de funcionalidade foi acessada, qual o texto digitado pelo usuário, a data e a hora da digitação, dentre outras.

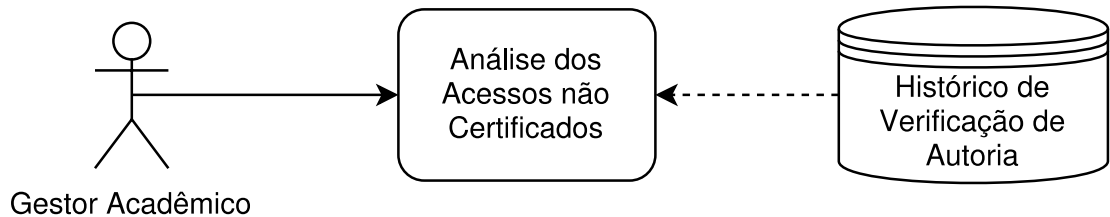

Figura 4: Terceira Fase do Mecanismo Proposto: Análise de Autorias. 
O módulo de Análise de Autorias permite, portanto, que os gestores do meio acadêmico possam, diante das informações fornecidas, identificar possíveis problemas junto aos usuários do AVA e decidir que providências tomar em cada caso.

Ao variar o intervalo do filtro de consulta deste módulo, os gestores responsáveis podem realizar diferentes análises longitudinais quanto à evolução dos percentuais de autorias suspeitas dos usuários. A decisão sobre como proceder junto a cada usuário deve ser norteada pela política pedagógica adotada em cada instituição/curso. Por exemplo, gestores acadêmicos podem optar por apenas acompanhar (sem nenhum contato), em novas atividades, usuários que tenham apresentado uma elevação pontual e isolada do percentual de autorias suspeitas em uma determinada atividade. Por outro lado, usuários com recorrentes e elevados percentuais de autoria suspeita podem ser contatados pelos gestores acadêmicos a fim que estes busquem um melhor diagnóstico das causas do problema e, em função dele, decidam como atuar em cada caso.

São exemplos de possíveis ações a serem tomadas: ofertas de reforço pedagógico direcionado aos conteúdos das atividades associadas às autorias suspeitas, possivelmente incluindo novas atividades a serem desenvolvidas, revisão técnica voltada ao ajuste ou mesmo à substituição do modelo de reconhecimento de usuário, notificação formal junto ao usuário quanto à necessidade de lisura do processo acadêmico, dentre outras.

Outra questão importante que pode ser considerada sob o ponto de vista pedagógico nas análises é quanto à natureza das atividades e dos conteúdos associados a percentuais de autoria suspeita mais elevados. Atividades e conteúdos com elevada incidência de autorias suspeitas junto a diferentes usuários podem ser consequência do fato de que os recursos pedagógicos disponíveis sobre os respectivos assuntos no AVA estejam se mostrando inadequados e os usuários estejam recorrendo a apoio externo indevido. Diante deste cenário, uma revisão voltada ao aprimoramento de tais recursos pode ser uma intervenção recomendável.

\section{Protótipo}

\subsection{Considerações Gerais}

A fim de demonstrar a viabilidade do mecanismo proposto neste artigo, optou-se por desenvolver um protótipo ${ }^{2}$ e integrá-lo a um AVA de código livre. Em função de sua popularidade, o AVA escolhido foi o Moodle.

Para extrair informações da digitação foi criada uma função de captura de dados em javascript (usando o YUI - Yahoo! User Interface (YUI, 2017)). Esta função observa, com precisão de milissegundo, os eventos de quando o usuário aperta a tecla e de quando ele a solta, utilizando a classe "capture_keystroke". Esta função é utilizada nos módulos de coleta de dados e verificação de autoria. Cabe ressaltar ainda que tal função não sofre influência da latência da rede, pois opera em memória, na máquina do usuário. Isso diminui as chances de ocorrerem interferências no padrão de digitação do usuário, evitando problemas na confecção de seu modelo de reconhecimento.

Para processamento dos dados foram implementados serviços web em Python 3.5.2 (Python, 2017) com auxílio do micro framework flask 0.11 (Flask, 2017). Outrossim, foi utilizado a API scikit-learn versão 0.18 (Scikit-learn, 2017) para criar os modelos de reconhecimento. Para o armazenamento dos dados foi utilizado o sistema gerenciador de bancos de dados relacionais MySq1 5.7.14 (MySq1, 2017).

\footnotetext{
2 Os códigos utilizados na confecção do protótipo podem ser obtidos em: https://github.com/MarcoASCruz/Keystroke-Dynamic-Moodle
} 
A seguir encontram-se comentários considerados relevantes na implementação de cada módulo do protótipo.

\subsection{Coleta de Dados}

A Figura 5 apresenta uma visão parcial da interface do módulo de coleta de dados. Cabe destacar que algumas respostas são solicitadas mais de uma vez, conforme recomendado em vários trabalhos relacionados à dinâmica da digitação (Costa et al., 2005; Cavalcanti, 2005; Darabseh \& Namin, 2014).

A função de captura de dados foi vinculada a cada um dos campos a serem preenchidos pelo usuário. Os dados brutos coletados são em seguida, enviados para o servidor para serem armazenados no banco de dados.

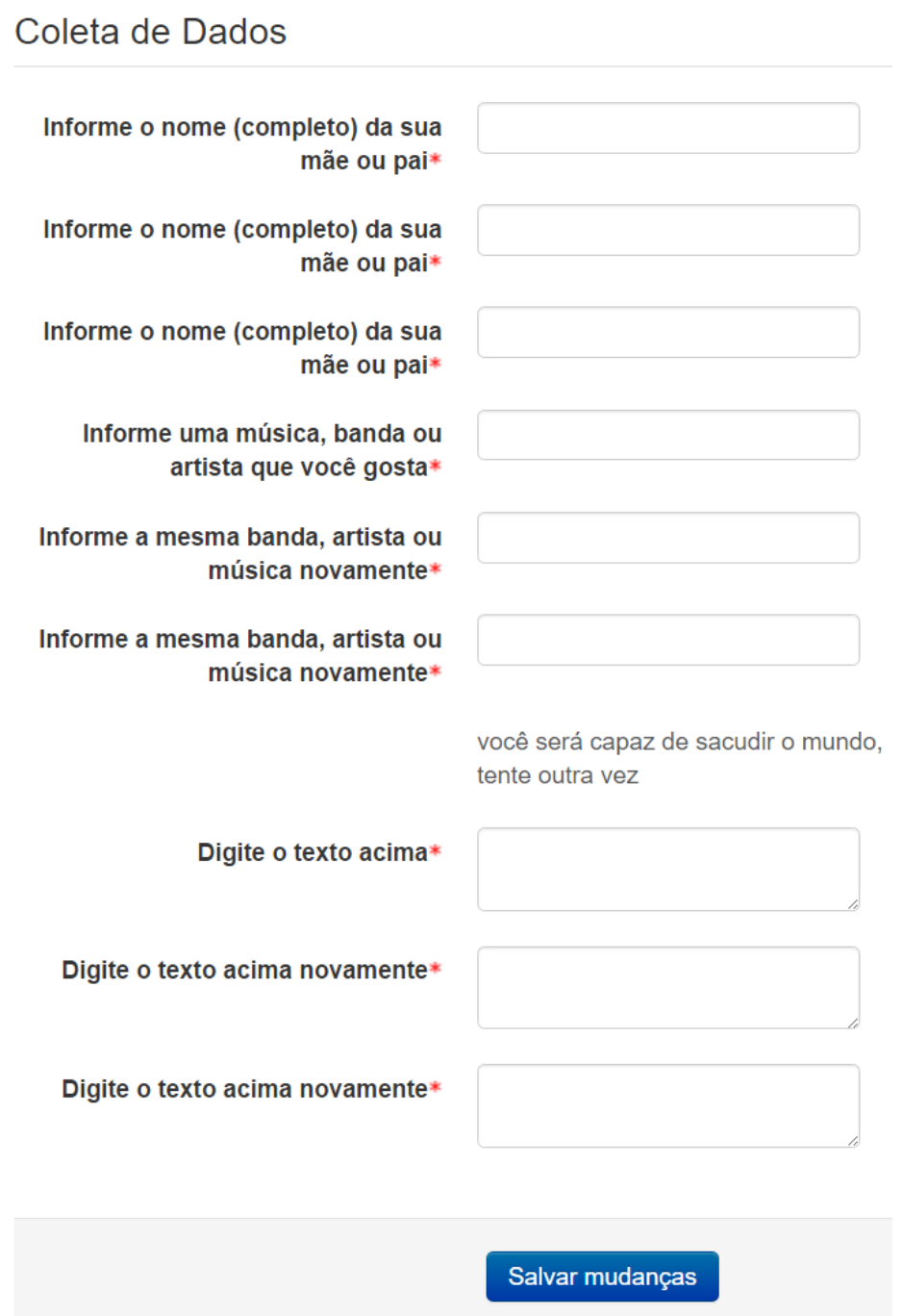

Figura 5: Formulário de Coleta de Dados.

\subsection{Construção do Modelo de Reconhecimento}

Para implementar o módulo de Construção do Modelo de Reconhecimento foram desenvolvidos dois subprogramas: o pré-processamento, que prepara os dados para serem utilizados pelos classificadores, e a construção do modelo do usuário, que compara diversos algoritmos de classificação com o intuito de selecionar o melhor modelo para o usuário. 
O pré-processamento foi implementando buscando seguir a descrição do mecanismo proposto, conforme descrito na Seção 4.2. Portanto, neste programa foram implementadas a extração das características, a construção de atributos e a normalização dos dados.

As características extraídas foram o tempo de pressionamento e a latência, da mesma maneira que foi exemplificada na Seção 2.1.

Os atributos construídos foram a média e o desvio padrão de cada característica para cada amostra, resultando em uma tabela como a da Figura 6.

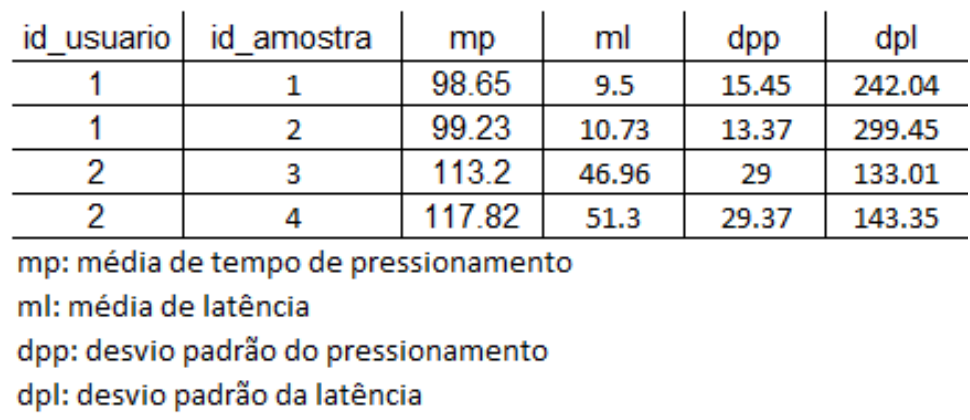

Figura 6: Tabela com os atributos construídos a partir dos dados brutos.

Por fim, foi feita a normalização linear (Han, Pei, \& Kamber, 2011) nos dados consolidados, mapeando os atributos em uma escala entre 0 e 1 (onde 1 corresponde ao valor máximo e 0 o valor mínimo de cada atributo coletado).

No protótipo desenvolvido, o programa de construção do modelo do usuário não foi integrado ao Moodle, devido ao tempo necessário para implementação de interfaces gráficas apropriadas ao processo. No entanto, o referido programa pode ser executado a partir da execução de serviços web criados com auxílio do microframework Flask. Os serviços foram codificados em linguagem Python e combinam funções disponíveis na biblioteca scikit-learn para executar a validação cruzada com os vários algoritmos apresentados na Seção 2.2 e identificar o melhor modelo de classificação gerado. Três saídas são produzidas, uma planilha com as acurácias dos modelos produzidos pelos algoritmos durante a validação cruzada, um arquivo contendo o melhor modelo de classificação gerado para o usuário analisado e um registro de dados que é inserido em uma tabela do banco de dados denominada "modelo do usuário". Esta tabela possui três campos:

- Identificação do usuário

- Identificação do algoritmo

- Identificação do caminho do diretório onde fica armazenado o arquivo que contém o melhor modelo gerado pelo processo.

\subsection{Verificação de Autoria}

A funcionalidade do Moodle escolhida para ser integrada ao protótipo do mecanismo proposto foi o Fórum. Sua escolha se deve basicamente ao fato de ser um dos recursos mais usados do ambiente e que permite a obtenção de amostras de texto de tamanho e conteúdo variáveis. A função de captura de dados foi vinculada aos campos assunto e mensagem do formulário de criação de tópico de discussão mostrados na Figura 7 e também ao campo de postagem de respostas aos tópicos de discussão. 


\section{Novo tópico de discussão}

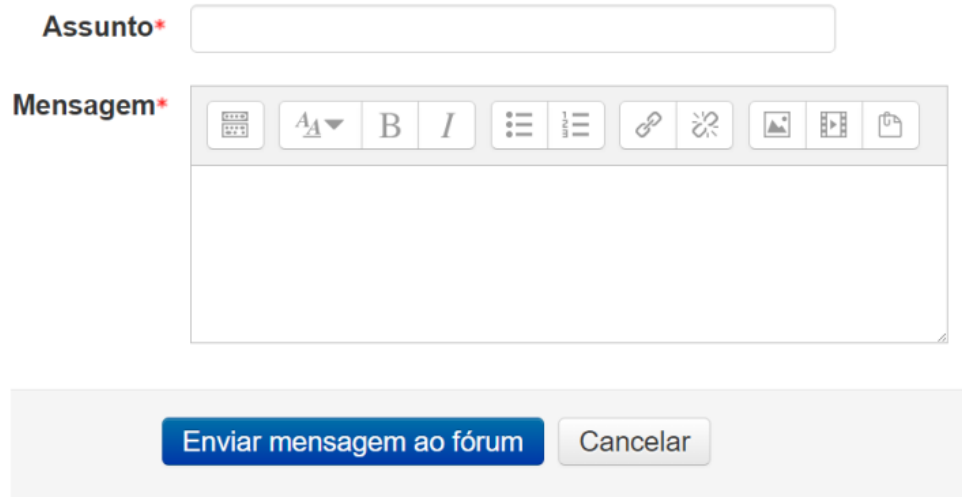

Figura 7: Formulário de Postagem de Assuntos do Fórum - os conteúdos dos campos assunto e mensagem são coletados pelo protótipo e submetidos ao reconhecimento de usuário.

Assim sendo, sempre que um usuário digita algum conteúdo em um desses campos, o padrão de digitação é coletado e, posteriormente submetido à avaliação do modelo de classificação correspondente. No protótipo, foi implementado um programa em Python que executa o processo de avaliação. Tal programa recupera a identificação do algoritmo de classificação e do modelo correspondente a partir de uma consulta à tabela "modelo de usuário", tomando como base o usuário autenticado no Moodle mediante login e senha no início da sessão de utilização do AVA. Em seguida, o programa interpreta e processa o modelo de classificação recuperado sobre o padrão do texto digitado, obtendo como resposta o resultado da análise da autoria: confirmada ou suspeita. Por fim, o programa salva o resultado e os dados correspondentes em uma tabela intitulada "histórico de verificação de autoria".

Cabe ressaltar que não foi necessário realizar nenhuma mudança nas interfaces originais do Moodle, sendo transparente para o usuário final a associação dos campos à função de captura de dados e ao acionamento do programa que recupera e aplica o modelo de classificação correspondente. Desta forma, o uso do mecanismo proposto se comporta de forma não intrusiva durante seu processamento.

\subsection{Análise de Autoria}

O protótipo deste módulo consiste basicamente da geração de relatórios extraídos a partir de consultas realizadas sobre os resultados das verificações de autoria previamente executadas. As figuras 8 e 9 ilustram dois desses relatórios. O relatório da Figura 8 apresenta os usuários em ordem decrescente de autorias suspeitas detectadas pelo sistema. Caso o gestor acadêmico deseje ver os detalhes dessas autorias, o relatório da Figura 9 pode ser consultado.

\begin{tabular}{|c|c|c|}
\hline \multicolumn{3}{|c|}{ Gestor Acadêmico: Gestor Teste } \\
\hline \multicolumn{3}{|c|}{ Período consultado: 1/07/2016 até 31/07/2016 } \\
\hline & $\%$ de Autorias & $\%$ de Autorias \\
\hline Usuário & Certificadas & Suspeitas \\
\hline B & $50 \%$ & $50 \%$ \\
\hline $\mathrm{C}$ & $90 \%$ & $10 \%$ \\
\hline A & $99 \%$ & $1 \%$ \\
\hline
\end{tabular}


Usuário: $\mathrm{B}$

$\%$ de autenticações suspeitas: $50 \%$

\begin{tabular}{llll} 
Funcionalidade & Referência & Data e Hora & Texto Digitado \\
\hline Fórum - Novo tópico de discussão & Título & $13 / 07 / 2016: 12: 32$ & Inteligência Artificial \\
\hline Fórum - Novo tópico de discussão & Conteúdo & $13 / 07 / 2016: 12: 32$ & Inteligência Artificial é uma área do conhecimento que estuda ... \\
\hline Fórum - Resposta & Conteúdo & $14 / 07 / 2016: 13: 00$ & As Redes Neurais podem ter várias camadas intermediáras e ...
\end{tabular}

Figura 9: Exemplo de Relatório - Detalhamento das Autenticações Suspeitas Associadas a um Usuário.

\section{Estudo de Caso}

A fim de avaliar a viabilidade do mecanismo proposto, o protótipo desenvolvido e integrado ao Moodle foi aplicado em uma disciplina de pós-graduação com dezessete usuários, sendo dois docentes e quinze estudantes, durante o mês de julho de 2016. Cabe ressaltar que a carga horária desta disciplina foi dividida igualmente em presencial e não presencial. Além disso, a prática na utilização do Moodle como ferramenta de apoio às atividades pedagógicas já fazia parte da cultura institucional.

A coleta de dados foi realizada na primeira semana. Para cada usuário, foram coletadas treze amostras, totalizando duzentas e vinte e uma amostras no conjunto de dados completo. Assim, considerando qualquer usuário, o conjunto de dados sempre dispôs de treze amostras positivas e duzentas e oito negativas.

Durante a segunda semana, 7 algoritmos de classificação (os mesmos mencionados na Seção 2.2) foram avaliados sobre o conjunto de dados coletado: o K-NN, Centroide Mais Próximo, SVM, Naïve Bayes, Árvore de Decisão, Floresta Aleatória e as Redes Neurais Artificiais.

No algoritmo K-NN foram utilizados 5 vizinhos próximos e foi considerado um peso maior para pontos mais próximos. O Centroide Mais Próximo utilizou a distância euclidiana.

O SVM usou como função-núcleo o RBF e a taxa de penalidade de erro igual a 0.5. Para o algoritmo Naïve Bayes o sckit-learning não oferecia parâmetros a serem configurados.

$\mathrm{Na}$ Árvore de Decisão, para escolher a função que mede a qualidade da divisão foi utilizado o Índice Gini e a estratégia de seleção feita na divisão de cada nó escolhia o melhor deles para ser selecionado.

No algoritmo Floresta Aletória foram configuradas nove árvores com os mesmos atributos da árvore de decisão anterior.

Nas Redes Neurais Artificiais foram utilizados 100 neurônios na camada intermediária, o algoritmo utilizado foi o adam (Kingma \& $\mathrm{Ba}, 2014$ ), o parâmetro de penalização foi igual a 0.01 e a taxa de aprendizado foi de 0.1 .

Para cada usuário, cada algoritmo foi avaliado em um processo de validação cruzada com 10 conjuntos. Os resultados do processo de avaliação para cada par (usuário, algoritmo) seguem expostos na Tabela 2. O melhor resultado obtido para cada usuário está destacado em negrito. A métrica de avaliação utilizada foi a acurácia dos modelos de reconhecimento. Cabe ressaltar que o pior desempenho da validação cruzada ficou em $92,44 \%$ de acurácia para os usuários 7, 8, 11, 14 e 17. Em todos os demais casos, o melhor resultado foi igual ou superior a 
este percentual, o que caracteriza um desempenho global competitivo em relação aos obtidos por trabalhos similares.

A segunda fase do processamento do mecanismo proposto foi executada durante a segunda quinzena do mês de julho. Durante esse período, para realização de suas atividades não presenciais, os usuários do Moodle acessaram, dentre outras, as funcionalidades do fórum. $\mathrm{O}$ mecanismo proposto verificou, para cada usuário, a autoria de suas postagens, registrando os resultados da verificação. Durante a primeira semana do mês de agosto de 2016, os docentes da disciplina tiveram acesso aos relatórios gerados pelo módulo de Análise de Autorias.

A Figura 10 apresenta, a título ilustrativo, uma visão sintética dos resultados obtidos com o mecanismo proposto no estudo de caso realizado. Cabe ressaltar que os baixos percentuais de autorias suspeitas refletiram o comportamento geral dos usuários do estudo de caso. Para simular autorias suspeitas foi pedido para 4 usuários $(9,10,11$ e 12) que estes deixassem que outras pessoas digitassem em seu lugar em alguns momentos. Como esperado, tais casos se refletiram levando à identificação desses usuários como aqueles com maiores percentuais de autorias suspeitas.

Apesar de eventuais imprecisões dos modelos em reconhecer corretamente a autoria de todos os textos digitados, foi possível observar por meio do protótipo implementado e do estudo de caso realizado que o mecanismo possui aplicação viável na autenticação periódica e não intrusiva de usuários de AVAs.

Tabela 2: Resultados da Validação Cruzada com 10 Conjuntos em termos de Média e Desvio Padrão (\%)

\begin{tabular}{|c|c|c|c|c|c|c|c|}
\hline Usuários & K-NN & $\begin{array}{c}\text { Centroide } \\
\text { Mais } \\
\text { Próxima }\end{array}$ & SVM & $\begin{array}{l}\text { Naïve } \\
\text { Bayes }\end{array}$ & $\begin{array}{l}\text { Árvore de } \\
\text { Decisão }\end{array}$ & $\begin{array}{c}\text { Floresta } \\
\text { Aleatória }\end{array}$ & $\begin{array}{c}\text { Redes } \\
\text { Neurais } \\
\text { Artificiais }\end{array}$ \\
\hline 1 & $\begin{array}{c}95,11 \pm \\
3,05\end{array}$ & $\begin{array}{c}78,67 \pm \\
15,97\end{array}$ & $\begin{array}{c}94,22 \pm \\
1,82\end{array}$ & $\begin{array}{c}94,22 \pm \\
2,72\end{array}$ & $\begin{array}{c}91,11 \pm \\
3,97\end{array}$ & $\begin{array}{c}96,00 \pm \\
3,13\end{array}$ & $\begin{array}{c}93,33 \pm \\
2,91\end{array}$ \\
\hline 2 & $\begin{array}{c}97,33 \pm \\
3,48\end{array}$ & $\begin{array}{c}83,56 \pm \\
9,52\end{array}$ & $\begin{array}{c}94,22 \pm \\
1,82\end{array}$ & $\begin{array}{c}96,00 \pm \\
3,56\end{array}$ & $\begin{array}{c}94,22 \pm \\
3,83\end{array}$ & $\begin{array}{c}95,11 \pm \\
4,04\end{array}$ & $\begin{array}{c}94,22 \pm \\
1,82\end{array}$ \\
\hline 3 & $\begin{array}{c}92,89 \pm \\
4,83\end{array}$ & $\begin{array}{c}64,44 \pm \\
13,89\end{array}$ & $\begin{array}{c}94,22 \pm \\
1,82\end{array}$ & $\begin{array}{c}92,44 \pm \\
4,36\end{array}$ & $\begin{array}{c}90,22 \pm \\
5,11\end{array}$ & $\begin{array}{c}95,11 \pm \\
2,29\end{array}$ & $\begin{array}{c}93,33 \pm \\
2,91\end{array}$ \\
\hline 4 & $\begin{array}{c}96,00 \pm \\
2,32\end{array}$ & $\begin{array}{c}87,56 \pm \\
11,31\end{array}$ & $\begin{array}{c}94,67 \pm \\
3,68\end{array}$ & $\begin{array}{c}95,56 \pm \\
3,87\end{array}$ & $\begin{array}{c}93,33 \pm \\
6,92\end{array}$ & $\begin{array}{c}96,89 \pm \\
3,35\end{array}$ & $\begin{array}{c}94,67 \pm \\
3,73\end{array}$ \\
\hline 5 & $\begin{array}{c}94,67 \pm \\
3,12\end{array}$ & $\begin{array}{c}66,67 \pm \\
15,00\end{array}$ & $\begin{array}{c}94,22 \pm \\
1,82\end{array}$ & $\begin{array}{c}92,44 \pm \\
4,39\end{array}$ & $\begin{array}{c}90,67 \pm \\
5,73\end{array}$ & $\begin{array}{c}95,56 \pm \\
3,90\end{array}$ & $\begin{array}{c}92,44 \pm \\
6,25\end{array}$ \\
\hline 6 & $\begin{array}{c}93,78 \pm \\
5,05\end{array}$ & $\begin{array}{c}70,67 \pm \\
14,58\end{array}$ & $\begin{array}{c}94,22 \pm \\
1,82\end{array}$ & $\begin{array}{c}93,33 \pm \\
3,86\end{array}$ & $\begin{array}{c}93,33 \pm \\
2,85\end{array}$ & $\begin{array}{c}95,11 \pm \\
3,54\end{array}$ & $\begin{array}{c}93,78 \pm \\
2,77\end{array}$ \\
\hline 7 & $\begin{array}{c}93,33 \pm \\
2,09\end{array}$ & $\begin{array}{c}72,44 \pm \\
18,50\end{array}$ & $\begin{array}{c}94,22 \pm \\
1,82\end{array}$ & $\begin{array}{c}92,89 \pm \\
2,08\end{array}$ & $\begin{array}{c}85,33 \pm \\
9,44\end{array}$ & $\begin{array}{c}92,44 \pm \\
4,00\end{array}$ & $\begin{array}{c}94,22 \pm \\
1,82\end{array}$ \\
\hline 8 & $\begin{array}{c}92,44 \pm \\
4,39\end{array}$ & $\begin{array}{c}67,11 \pm \\
14,08\end{array}$ & $\begin{array}{c}94,22 \pm \\
1,82\end{array}$ & $\begin{array}{c}94,22 \pm \\
1,82\end{array}$ & $\begin{array}{c}87,56 \pm \\
5,38\end{array}$ & $\begin{array}{c}92,89 \pm \\
2,08\end{array}$ & $\begin{array}{c}93,78 \pm \\
2,01\end{array}$ \\
\hline 9 & $\begin{array}{c}92,44 \pm \\
4,32\end{array}$ & $\begin{array}{c}72,44 \pm \\
13,11\end{array}$ & $\begin{array}{c}94,22 \pm \\
1,82\end{array}$ & $\begin{array}{c}94,67 \pm \\
3,73\end{array}$ & $\begin{array}{c}92,44 \pm \\
2,90\end{array}$ & $\begin{array}{c}93,33 \pm \\
2,92\end{array}$ & $\begin{array}{c}91,56 \pm \\
6,04\end{array}$ \\
\hline 10 & $\begin{array}{c}91,56 \pm \\
3,80\end{array}$ & $\begin{array}{c}78,67 \pm \\
14,03\end{array}$ & $\begin{array}{c}94,22 \pm \\
1,82\end{array}$ & $\begin{array}{c}94,67 \pm \\
3,29\end{array}$ & $\begin{array}{c}94,67 \pm \\
4,40\end{array}$ & $\begin{array}{c}95,11 \pm \\
5,44\end{array}$ & $\begin{array}{c}92,89 \pm \\
5,40\end{array}$ \\
\hline 11 & $\begin{array}{c}93,33 \pm \\
3,64\end{array}$ & $\begin{array}{c}68,44 \pm \\
12,34\end{array}$ & $\begin{array}{c}94,22 \pm \\
1,82\end{array}$ & $\begin{array}{c}90,67 \pm \\
5,41\end{array}$ & $\begin{array}{c}92,44 \pm \\
5,45\end{array}$ & $\begin{array}{c}92,44 \pm \\
4,10\end{array}$ & $\begin{array}{c}92,00 \pm \\
5,23\end{array}$ \\
\hline
\end{tabular}




\begin{tabular}{|c|c|c|c|c|c|c|c|}
\hline \multirow{2}{*}{12} & $95,56 \pm$ & $81,78 \pm$ & $94,22 \pm$ & $\mathbf{9 6 , 8 9} \pm$ & $96,00 \pm$ & $95,11 \pm$ & $88,00 \pm$ \\
& 6,34 & 13,51 & 1,82 & $\mathbf{4 , 4 6}$ & 6,20 & 6,83 & 9,07 \\
& $94,67 \pm$ & $84,44 \pm$ & $94,22 \pm$ & $\mathbf{9 5 , 5 6} \pm$ & $93,78 \pm$ & $93,78 \pm$ & $93,78 \pm$ \\
\multirow{2}{*}{13} & 2,50 & 11,76 & 1,82 & $\mathbf{4 , 3 1}$ & 4,03 & 2,13 & 4,02 \\
& $91,56 \pm$ & $46,22 \pm$ & $\mathbf{9 2 , 4 4} \pm$ & $92,00 \pm$ & $82,67 \pm$ & $88,44 \pm$ & $88,44 \pm$ \\
& 2,50 & 15,82 & $\mathbf{1 , 8 1}$ & 3,36 & 8,53 & 6,20 & 9,64 \\
\hline \multirow{2}{*}{14} & $95,11 \pm$ & $78,67 \pm$ & $94,22 \pm$ & $93,78 \pm$ & $93,33 \pm$ & $\mathbf{9 5 , 5 6} \pm$ & $92,44 \pm$ \\
& 5,06 & 16,05 & 1,82 & 2,13 & 4,08 & $\mathbf{3 , 4 5}$ & 3,46 \\
\hline \multirow{2}{*}{15} & $96,89 \pm$ & $93,33 \pm$ & $96,44 \pm$ & $96,00 \pm$ & $94,67 \pm$ & $\mathbf{9 7 , 3 3} \pm$ & $92,44 \pm$ \\
& 2,80 & 5,63 & 2,76 & 3,21 & 4,85 & $\mathbf{2 , 2 1}$ & 4,56 \\
\hline \multirow{2}{*}{16} & $92,89 \pm$ & $62,22 \pm$ & $\mathbf{9 4 , 2 2} \pm$ & $92,44 \pm$ & $87,11 \pm$ & $89,78 \pm$ & $92,89 \pm$ \\
& 2,14 & 17,83 & $\mathbf{1 , 8 2}$ & 3,40 & 8,90 & 7,23 & 2,89 \\
\hline \multirow{2}{*}{17} & & & & & & & \\
\end{tabular}

\begin{tabular}{|c|c|c|}
\hline Usuário & $\begin{array}{l}\% \text { de Autorias } \\
\text { Certificadas }\end{array}$ & $\begin{array}{l}\% \text { de Autorias } \\
\text { Suspeitas }\end{array}$ \\
\hline 12 & $2.7 \%$ & $97.3 \%$ \\
\hline 10 & $2.9 \%$ & $97.1 \%$ \\
\hline 9 & $4.5 \%$ & $95.5 \%$ \\
\hline 11 & $5.3 \%$ & $94.7 \%$ \\
\hline 13 & $91.2 \%$ & $8.8 \%$ \\
\hline 17 & $92.5 \%$ & $7.5 \%$ \\
\hline 14 & $92.9 \%$ & $7.1 \%$ \\
\hline 6 & $93.3 \%$ & $6.7 \%$ \\
\hline 15 & $94.6 \%$ & $5.4 \%$ \\
\hline 7 & $94.8 \%$ & $5.2 \%$ \\
\hline 3 & $95.6 \%$ & $4.4 \%$ \\
\hline 8 & $96.0 \%$ & $4.0 \%$ \\
\hline 4 & $96.2 \%$ & $3.8 \%$ \\
\hline 1 & $97.3 \%$ & $2.7 \%$ \\
\hline 5 & $97.6 \%$ & $2.4 \%$ \\
\hline 16 & $98.5 \%$ & $1.5 \%$ \\
\hline 2 & $99.1 \%$ & $0.9 \%$ \\
\hline
\end{tabular}

Figura 10: Relatório com Resultados da Verificação de Autenticidade.

Além da avaliação do desempenho dos modelos de reconhecimento construídos neste estudo de caso, também foi realizada uma análise quanto à usabilidade das interfaces e às possibilidades de ações pedagógicas oferecidas pelo protótipo implementado. Esta análise teve como base os resultados obtidos por meio de uma pesquisa qualitativa aplicada junto aos usuários do ambiente após o período de utilização do protótipo. Tal pesquisa foi elaborada a partir das heurísticas de usabilidade de interface propostas por Nielsen (1993), considerando a experiência prévia do público alvo na utilização do Moodle. Foi composta por questões 
fechadas com três respostas cada, segundo a escala likert ${ }^{3}$. A ferramenta utilizada para a realização da pesquisa foi o GoogleForms ${ }^{\circledR}$. Apenas o módulo de construção do modelo de conhecimento não foi avaliado por meio da pesquisa mencionada, devido ao fato de ser o único não integrado ao Moodle.

Quatorze dos dezessete usuários responderam às questões referentes aos módulos de coleta de dados e de verificação de autoria enquanto apenas dois (os docentes) responderam às questões sobre o módulo de análise de autoria. Cabe ressaltar que embora o módulo de verificação de autoria não seja acessado diretamente e de forma transparente pelos usuários, optou-se por incluí-lo na pesquisa pelo fato do mesmo ter sido integrado ao Fórum, funcionalidade do Moodle utilizada no estudo de caso. Cabe ressaltar também que, no caso do módulo de análise de autoria, foram incluídas questões abertas a fim de permitir que os docentes expressassem suas opiniões acerca das funcionalidades e das possibilidades de ações pedagógicas oferecidas pelo módulo. Os gráficos das figuras 11 e 12 apresentam os resultados apurados sobre cada módulo.

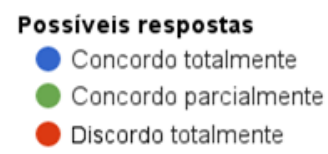

As interfaces utilizadas seguiram o mesmo padrão adotado pelo Moodle.

Concordo parcialmente $14,3 \%$

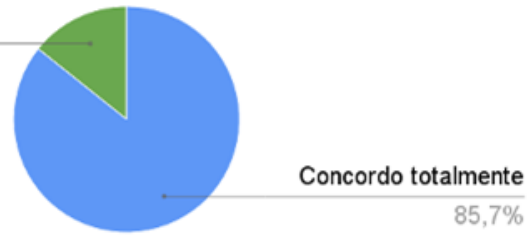

Foi fácil compreender as interfaces, não havendo necessidade de explicações adicionais sobre como utilizá-las.

Concordo parcialmente $14,3 \%$

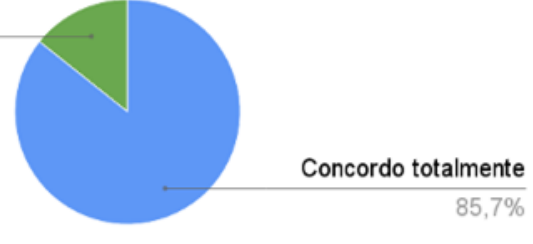

Comparando com minha experiência anterior de utilização do Moodle, não houve diferença no tempo de digitação das respostas nos campos solicitados e nem no tempo de gravação dos dados digitados.

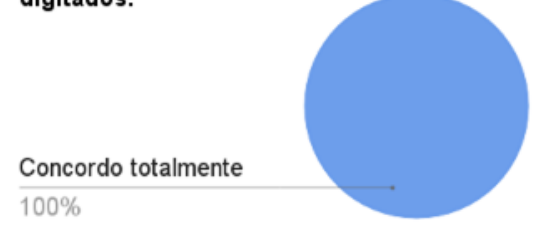

Figura 11: Análise de Interface da Coleta de Dados.

3 O instrumento de pesquisa de usabilidade utilizado neste estudo pode ser acessado em: https://drive.google.com/open?id=0B5hbH38Q0EmuUjN0ZmhTVnVCUjQ 


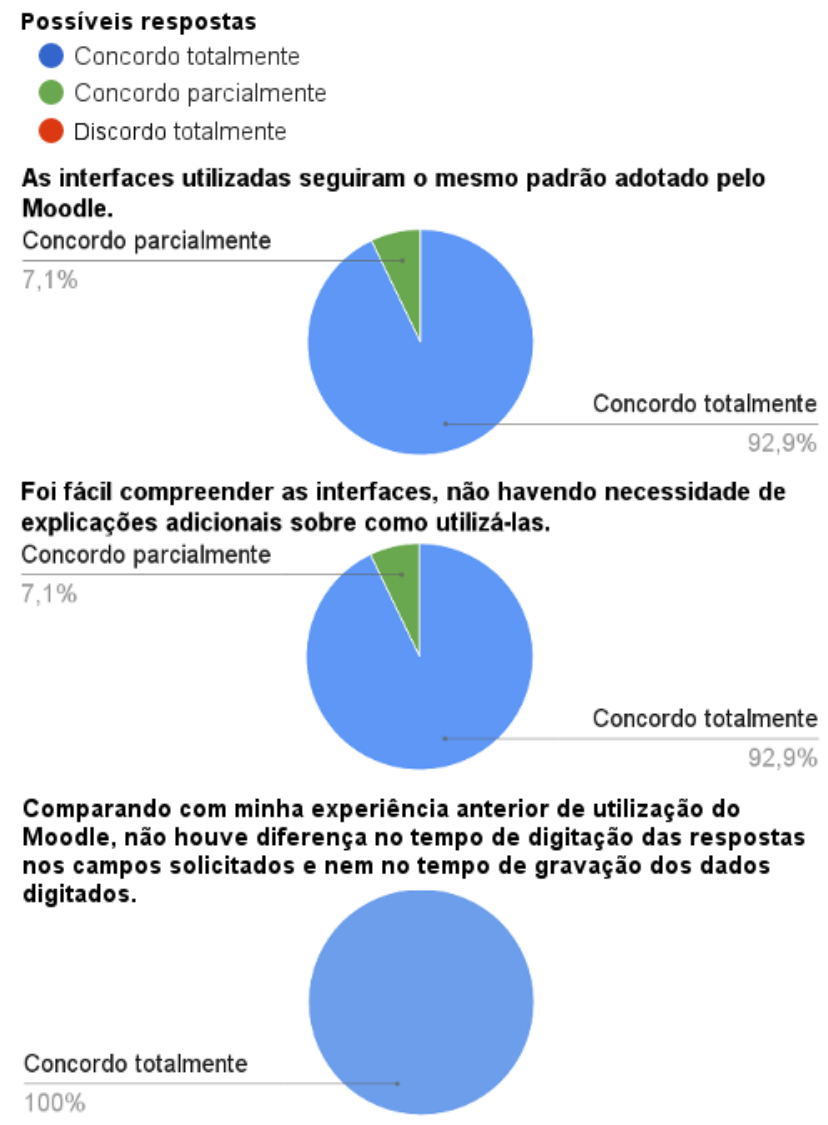

Figura 12: Análise de Interface da Verificação de Autoria.

Os usuários foram unânimes em concordar que as interfaces dos módulos de coleta de dados e de verificação de autoria seguiram o mesmo padrão adotado pelo Moodle. De fato, a compatibilização das interfaces do protótipo com o AVA esta foi uma das premissas adotadas na implementação. De forma análoga, os entrevistados afirmaram ter sido fácil o entendimento sobre como utilizar os referidos módulos. E, por fim, os usuários não perceberam diferença de tempo de resposta entre a utilização das interfaces do protótipo e as interfaces originais do Moodle.

Com relação às respostas dos docentes sobre as perguntas abertas, ambos concordaram que o protótipo do módulo de Análise de Autoria, embora limitado em suas funcionalidades, apresenta alguns recursos para identificar possíveis problemas de autenticidade de autoria. $\mathrm{Na}$ opinião dos docentes, o protótipo do módulo não é suficiente para apoiar a viabilização de uma política pedagógica de combate a fraudes relacionadas à autoria de atividades a distância. Segundo eles, para ter condições de apoiar tal política, novas funcionalidades deveriam ser desenvolvidas. Um exemplo interessante citado foi a construção de um canal de comunicação entre gestores acadêmicos e os usuários do AVA para tratar de questões relacionadas à autoria de atividades desenvolvidas a distância na plataforma. Por meio da utilização deste canal seria construída uma memória das interações entre gestores e usuários e das providências tomadas em cada caso. Futuramente essa memória poderia ser utilizada para aprimorar os modelos de reconhecimento de usuário do AVA. Outra sugestão de destaque foi a introdução dos conceitos de certificação de autoria automática e certificação de autoria manual. A primeira seria utilizada para as certificações realizadas automaticamente pelo sistema enquanto que a segunda seria informada pelos gestores acadêmicos em consequência dos contatos realizados junto aos usuários. Este tipo de informação também poderia oferecer subsídios futuros para $o$ aprimoramento dos modelos de reconhecimento do sistema. 


\section{Considerações Finais}

Apesar dos avanços que vêm sendo proporcionados pelos ambientes virtuais de aprendizagem (AVAs), um dos principais desafios da Educação a distância é quanto à autenticidade dos usuários durante o período em que utilizam esses ambientes. A abordagem em geral adotada nos AVAs permite que usuários não credenciados, após a autenticação inicial, assumam o papel de usuários credenciados, o que pode ocasionar diversos problemas de segurança e de natureza pedagógica, dentre outras. Neste cenário, o objetivo deste trabalho foi propor um mecanismo que realizasse a autenticação periódica não intrusiva de usuários em AVAs. O mecanismo proposto utiliza técnicas de aprendizado de máquina para construir modelos de reconhecimento baseados na dinâmica da digitação dos usuários e é independente de AVA, podendo ser integrado às diferentes plataformas existentes. Um protótipo do mecanismo proposto foi implementado, integrado ao Moodle, e aplicado em uma situação real de utilização deste AVA. Cabe ressaltar que, embora alguns modelos do estudo de caso tenham apresentado desempenhos um pouco inferiores $(92,44 \%)$ aos reportados em alguns trabalhos da área $(97,0 \%)$, ainda assim o estudo realizado constitui-se em um indicador favorável à viabilidade de aplicação do mecanismo proposto. Dentro desta perspectiva, o aprimoramento do desempenho dos modelos construídos no estudo de caso pode ser objeto de trabalhos futuros.

Entre outras alternativas de trabalhos futuros, as seguintes principais iniciativas podem ser destacadas: ampliação e diversificação do público alvo da experimentação do protótipo desenvolvido; realização de experimentos com maior volume de usuários e cursos; aprofundamento da avaliação dos aspectos de usabilidade do protótipo desenvolvido; incorporação de novas funcionalidades no módulo de análise de autorias que apoiem os gestores acadêmicos na escolha e no acompanhamento das providências a serem tomadas diante dos resultados apresentados pelo módulo e das análises realizadas; refinamento do protótipo visando a disponibilização de um plug-in de código aberto para o Moodle; e a investigação de alternativas tanto de algoritmos de aprendizado de máquina quanto de características a serem extraídas a partir da dinâmica da digitação.

\section{References}

Ali, M. M., Mahale, V. H., Yannawar, P., \& Gaikwad, A. T. (2016). Overview of fingerprint recognition system. In Electrical, Electronics, and Optimization Techniques (ICEEOT), International Conference on (pp. 1334-1338). IEEE. doi: 10.1109/ICEEOT.2016.7754900 [GS Search]

Alsultan, A., \& Warwick, K. (2013). Keystroke dynamics authentication: a survey of free-text methods. In International Journal of Computer Science Issues, 10(4), 1-10. [GS Search]

Araújo, L. C., Sucupira, L. H., Lizarraga, M. G., Ling, L. L., \& Yabu-Uti, J. B. T. (2005). User authentication through typing biometrics features. IEEE transactions on signal processing, 53(2), 851-855. doi: 10.1109/TSP.2004.839903 [GS Search]

Banerjee, S. P., \& Woodard, D. L. (2012). Biometric authentication and identification using keystroke dynamics: A survey. Journal of Pattern Recognition Research, 7(1), 116-139. doi: $\underline{10.13176 / 11.427}$ [GS Search]

Bhatt, S., \& Santhanam, T. (2013). Keystroke dynamics for biometric authentication-A survey. In Pattern Recognition, Informatics and Mobile Engineering (PRIME), 2013 International Conference on (pp. 17-23). IEEE. doi: 10.1109/ICPRIME.2013.6496441 [GS Search] 
Boechat, G. C., Ferreira, J. C., \& Carvalho Filho, E. C. (2007). Authentication personal. In Intelligent and Advanced Systems, 2007. ICIAS 2007. International Conference on (pp. 254256). IEEE. doi: 10.1109/ICIAS.2007.4658385 [GS Search]

Bowyer, K. W., Hollingsworth, K. P., \& Flynn, P. J. (2013). A survey of iris biometrics research: 2008-2010. In Handbook of iris recognition (pp. 15-54). Springer London. doi: 10.1007/978-1-4471-4402-1_2 [GS Search]

Breiman, L. (2001). Random forests. In Machine learning, 45(1), 5-32. doi: $\underline{\text { 10.1023/A:1010933404324 [GS Search] }}$

Bucci, M. A. M., \& da Silva Meneghel, P. (2008). Tecnologias e ferramentas gratuitas da Internet e sua aplicação aos programas de aprimoramento profissional à distância de equipes em bibliotecas universitárias. RBBD - Revista Brasileira de Biblioteconomia e Documentação, 4(2), 52-63. [GS Search]

Cavalcanti, G. D. C. (2005), Composição de biometria para sistemas multimodais de verificação de identidade pessoal (Tese de Doutorado), Universidade Federal de Pernambuco.

Clark, R. C., \& Mayer, R. E. (2016). E-learning and the science of instruction: Proven guidelines for consumers and designers of multimedia learning. In John Wiley \& Sons (pp. 10-15). doi: 10.1002/9781118255971 [GS Search]

Cortes, C., \& Vapnik, V. (1995). Support-vector networks. In Machine learning, 20(3), 273297. doi: $\underline{10.1007 / \mathrm{BF} 00994018 \text { [GS Search] }}$

Costa, C. R. D. N., Yared, G. F., Rodrigues, R. N., Yabu-Uti, J. B., Violaro, F., \& Ling, L. L. (2005). Autenticação Biométrica via Dinâmica da Digitação em Teclados Numéricos. In XXII Simpósio Brasileiro de Telecomunicações-SBrT’05 (pp. 04-08). [GS Search]

Cover, T., \& Hart, P. (1967). Nearest neighbor pattern classification. IEEE transactions on information theory, 13(1), 21-27. doi: 10.1109/TIT.1967.1053964 [GS Search]

Curtin, M., Tappert, C., Villani, M., Ngo, G., Simone, J., Fort, H. S., \& Cha, S. (2006). Keystroke biometric recognition on long-text input: A feasibility study. In Proc. Int. MultiConf. Engineers \& Computer Scientists (IMECS). [GS Search]

Darabseh, A., \& Namin, A. S. (2014). The accuracy of user authentication through keystroke features using the most frequent words. In Proceedings of the 9th Annual Cyber and Information Security Research Conference (pp. 85-88). ACM. doi: $\underline{10.1145 / 2602087.2602102}$ [GS Search]

Daugman, J. (2004). How iris recognition works. In IEEE Transactions on circuits and systems for video technology, 14(1), 21-30. doi: 10.1109/TCSVT.2003.818350 [GS Search]

Dehnavi, M. K., Sharafi, S. M., \& Nematbakhsh, N. (2011). Developing a e-learning model for tracking the continuous attendance of the students. In Journal of Theoretical and Applied Information Technology, 24(1), 62-68 [GS Search]

Diniz, F. A., Neto, F. M. M., Júnior, F. D. C. L., \& Fontes, L. M. D. O. (2012). RedFace: Um Sistema de Reconhecimento Facial para Identificação de Estudantes em um Ambiente Virtual de Aprendizagem. In RENOTE, 10(3) [GS Search]

Dowland, P. S., Furnell, S. M., \& Papadaki, M. (2002). Keystroke analysis as a method of advanced user authentication and response. In Security in the Information Society (pp. 215226). Springer US. doi: 10.1007/978-0-387-35586-3 17 [GS Search]

ECMA (2015). ECMAScript ${ }^{\circledR} 2015$ Language Specification. Retrieved from http://www.ecmainternational.org/ecma-262/6.0/ 
Faceli, K., Lorena, A. C., Gama, J., \& Carvalho, A. C. P. L. F. (2011). Inteligência Artificial: Uma abordagem de aprendizado de máquina. Rio de Janeiro: LTC, 2, 192.

Fayyad, U. M., \& Irani, K. B. (1992). The attribute selection problem in decision tree generation. In $A A A I$ (pp. 104-110). [GS Search]

Flask (2017). Flask Documentation. Retrieved from http://flask.pocoo.org/docs/0.11/

Galafassi, F. P., Gluz, J. C., \& Galafassi, C. (2013). Análise crítica das pesquisas recentes sobre as tecnologias de objetos de aprendizagem e ambientes virtuais de aprendizagem. Revista Brasileira de Informática na Educação, 21(3), 41-52. doi: 10.5753/RBIE.2013.21.03.100. [GS Search]

Goldschmidt, R., Bezerra, E., \& Passos, E. (2015). Data mining: conceitos, técnicas, algoritmos, orientações e aplicações. Elsevier, Brasil.

Gunetti, D., \& Picardi, C. (2005). Keystroke analysis of free text. ACM Transactions on Information and System Security (TISSEC), 8(3), 312-347. doi: 10.1145/1085126.1085129 [GS Search]

Gunetti, D., \& Ruffo, G. (1999). Intrusion detection through behavioral data. In International Symposium on Intelligent Data Analysis (pp. 383-394). Springer Berlin Heidelberg. doi: 10.1007/3-540-48412-4_32 [GS Search]

Han, J., Pei, J., \& Kamber, M. (2011). Data mining: concepts and techniques. Elsevier.

INEP (2014). Censo da Educação Superior - Notas Estatísticas. Retrieved from http://download.inep.gov.br/educacao_superior/censo_superior/documentos/2015/notas_sob re o censo da educacao superior 2014.pdf

Jafri, R., \& Arabnia, H. R. (2009). A survey of face recognition techniques. In Jips, 5(2), 41-68. doi: $10.3745 /$ JIPS.2009.5.2.041 [GS Search]

Jain, A. K., Nandakumar, K., \& Ross, A. (2016). 50 years of biometric research: Accomplishments, challenges, and opportunities. Pattern Recognition Letters, 79, 80-105. doi: 10.1016/j.patrec.2015.12.013 [GS Search]

King, D. L., \& Case, C. J. (2014). E-cheating: Incidence and trends among college students. In Issues in Information Systems, 15(1), 20-27 [GS Search]

Kingma, D., \& Ba, J. (2014). Adam: A method for stochastic optimization. In arXiv preprint arXiv:1412.6980 (pp. 1-15) [GS Search]

Kohavi, R. (1995). A study of cross-validation and bootstrap for accuracy estimation and model selection. In Ijcai (Vol. 14, No. 2, pp. 1137-1145) [GS Search]

Langley, P., Iba, W., \& Thompson, K. (1992). An analysis of Bayesian classifiers. In Aaai (Vol. 90, pp. 223-228) [GS Search]

Maio, D., Maltoni, D., Cappelli, R., Wayman, J. L., \& Jain, A. K. (2002). FVC2000: Fingerprint verification competition. In IEEE Transactions on Pattern Analysis and Machine Intelligence, 24(3), 402-412. doi: 10.1109/34.990140 [GS Search]

Mandaji, M. (2012). O processo de colaboração nos trabalhos de coautoria em ambientes virtuais de aprendizagem. Revista Brasileira de Informática na Educação, 20(1), 50-52. doi: 10.5753/RBIE.2012.20.1.50 [GS Search]

Marais, Emil, Argles, David and von Solms, Basie (2006) Security Issues Specific to eAssessments. In 8th Annual Conference on WWW Applications [GS Search] 
Moini, A., \& Madni, A. M. (2009). Leveraging biometrics for user authentication in online learning: a systems perspective. In IEEE Systems Journal, 3(4), 469-476. doi: $\underline{10.1109 / J S Y S T .2009 .2038957}$ [GS Search]

Monrose, F., \& Rubin, A. D. (2000). Keystroke dynamics as a biometric for authentication. Future Generation computer systems, 16(4), 351-359. doi: 10.1016/S0167-739X(99)00059$\underline{\mathrm{X}}$ [GS Search]

Moten Jr, J., Fitterer, A., Brazier, E., Leonard, J., \& Brown, A. (2013). Examining Online College Cyber Cheating Methods and Prevention Measures. In Electronic Journal of Elearning, 11(2), 139-146 [ [GS Search]

MySql (2017). MySql 5.7 Reference Manual. Retrieved from https://dev.mysql.com/doc/refman/5.7/en/

Nielsen, J. (1993). Usability engineering. Morgan Kaufmann Publishers Inc. San Francisco, CA, USA

O'Gorman, L. (2003). Comparing passwords, tokens, and biometrics for user authentication. In Proceedings of the IEEE, 91(12), 2021-2040. doi: 10.1109/JPROC.2003.819611 [GS Search]

Padmanabhan, J., \& Johnson Premkumar, M. J. (2015). Machine learning in automatic speech recognition: A survey. In IETE Technical Review, 32(4), 240-251. doi: $\underline{10.1080 / 02564602.2015 .1010611}$ [GS Search]

Parkhi, O. M., Vedaldi, A., \& Zisserman, A. (2015). Deep Face Recognition. In BMVC (Vol. 1, No. 3, p. 6) [GS Search]

Penteado, B. E., \& Marana, A. N. (2008). Autenticação biométrica on-line de usuários em aplicações web de Ensino a distância. In Companion Proceedings of the XIV Brazilian Symposium on Multimedia and the Web (pp. 53-56). ACM. doi: 10.1145/1809980.1809995 [GS Search]

Poersch, A. M., Santos, N. S., \& Nelson, M. A. V (2006). Estudo quantitativo da manutenção evolutiva em dois sistemas de código aberto. In III Workshop de Manutenção de Software Moderna - WMSWM (pp. 98-105) [GS Search]

Python (2017). Sobre o Python. Retrieved from https://www.python.org/about/

Rabuzin, K., Baca, M., \& Sajko, M. (2006). E-learning: Biometrics as a Security Factor. In Computing in the Global Information Technology, 2006. ICCGI'06. International MultiConference IEEE on (pp. 64-64). doi: 10.1109/ICCGI.2006.28 [GS Search]

Rolim, A. L., \& Bezerra, E. P. (2008). Um sistema de identificação automática de faces para um ambiente virtual de ensino e aprendizagem. In Companion Proceedings of the XIV Brazilian Symposium on Multimedia and the Web (pp. 129-132). ACM. doi: $\underline{10.1145 / 1809980.1810015}$ [GS Search]

Rosenblatt, F. (1962). Principles of neurodynamics. Spartan Books, New York

Salzberg, S. L. (1994). C4. 5: Programs for machine learning by j. ross quinlan. morgan kaufmann publishers, inc., 1993. In Machine Learning, 16(3), 235-240. doi: $\underline{10.1007 / \mathrm{BF} 00993309}$ [GS Search]

Scikit-learn (2017). Documentation of scikit-learn 0.18. Retrieved from http://scikitlearn.org/0.18/documentation.html

Teh, P. S., Teoh, A. B. J., \& Yue, S. (2013). A survey of keystroke dynamics biometrics. The Scientific World Journal. doi: 10.1155/2013/408280 [GS Search] 
Tibshirani, R., Hastie, T., Narasimhan, B., \& Chu, G. (2002). Diagnosis of multiple cancer types by shrunken centroids of gene expression. Proceedings of the National Academy of Sciences, 99(10), 6567-6572. doi: 10.1073/pnas.082099299 [GS Search]

Violettas, G. E., Theodorou, T. L., \& Stephanides, G. C. (2013). E-Learning Software Security: Tested for Security Vulnerabilities \& Issues. In e-Learning" Best Practices in Management, Design and Development of e-Courses: Standards of Excellence and Creativity", 2013 Fourth International Conference on (pp. 233-240). IEEE. doi: $\underline{10.1109 / E C O N F .2013 .66}$ [GS Search]

YUI (2017). YUI Documentation. Retrieved from https://github.com/yui/yui3/wiki/YUIDocumentation 